were used to determine the neurofibrillary tangle formation and $A B$ amyloid deposition in the hippocampal formation and inferior temporal gyrus in 36 Down's syndrome cases, aged 4 to 73 years. Neuropathological material was collected from several sources in Great Britain and America, and results are reported from the Massachusetts General Hospital, Boston, and the University of Manchester, England. Neurofibrillary tangles (NFTs) accumulated in patients with Down's syndrome over the age range 35 to 75 years, in the same anatomic locale as individuals with sporadic Alzheimer's disease. The entorhinal cortex, area CA1/subiculum, and other hippocampal subfields were especially vulnerable. Amyloid deposition is more widespread, accumulating over the years 30 to 50, and then reaching a plateau. Inheritance of the apolipoprotein E (Apo E) e4 genotype predisposed to more than double the amount of amyloid burden and was associated with increased numbers of senile plaques in Down's syndrome individuals with Alzheimer's disease. (Hyman BT et al. Neuropathological changes in Down's syndrome hippocampal formation. Effect of age and apolipoprotein E genotype. Arch Neurol April 1995;52:373-378). (Reprints: Dr Bradley T Hyman, Neurology Service, Massachusetts General Hospital, Fruit Street, Boston, MA 02114).

COMMENT. In a study at Mount Sinai School of Medicine, NY, the University of Kentucky, and the University of Geneva, Switzerland, quantitative analyses of neuropathologic changes in cerebral cortex of 16 patients (aged 6 to 74 years) with Down's syndrome and in 10 elderly individuals with Alzheimer's disease showed a similar time course of neurofibrillary tangle formation. Older patients with Down's syndrome had more neurofibrillary tangles and senile plaques than patients with Alzheimer's disease. Amyloid deposition preceeded neurofibrillary tangle formation. (Hof PR et al. Age-related distribution of neuropathologic changes in the cerebral cortex of patients with Down's syndrome. Quantitative regional analysis and comparison with

Alzheimer's disease. Arch Neurol April 1995;52:379-391).

\title{
MIGRAINE HEADACHES
}

\section{PERSISTENT VISUAL PHENOMENA IN MIGRAINE}

Ten patients with migraine and persistent positive visual phenomena lasting months to years are reported from the University of Pennsylvania, Scheie Eye Institute, the Children's Hospital of Philadelphia, University of Miami Bascom Palmer Eye Institute, and the Cleveland Clinic, OH. Ages ranged from 9 to 67 years but visual phenomena were very similar in their simplicity, quality, and involvement of the entire visual field. They consisted of diffuse small particles, such as TV static, snow, lines of ants, dots, and rain. Treatment with various medications was of no benefit. (Liu GT et al. Persistent positive visual phenomena in migraine. Neurology April 1995;45:664-668). (Reprints: Dr GT Liu, Division of Neuro-Ophthalmology, Department of Neurology, Hospital of the University of Pennsylvania, 3400 Spruce Street, Philadelphia, PA 19104).

COMMENT. Visual processing in 12 migraineurs has been studied at the Mass General Hospital (Wray SH et al. Brain Feb 1995;118:25-35). An inherited abnormal threshold to visual stimuli is suggested. The high speed of the migraineur's brain in discerning a single target in lowlevel visual tasks is consistent with an oversensitivity to visual stimuli. 